\title{
Will Social Media Make or Break Medical Conferences?
}

Corresponding Author:

Dr. Damian Roland ${ }^{1,2} \quad$ dr98@le.ac.uk

Tel: 01162585835

Dr. Natalie May ${ }^{3}$,

Dr Rick Body ${ }^{3}$,

Prof Simon Carley ${ }^{3}$

Dr. Mark D. Lyttle $e^{4,5}$

1. SAPPHIRE Group, Health Sciences, University of Leicester, UK

2. Paediatric Emergency Medicine Leicester Academic Group (PEMLA)

Emergency Department, Leicester Royal Infirmary, Leicester, UK

3. Emergency Department, Central Manchester University Hospitals NHS Foundation Trust, Cobbett House, Manchester Royal Infirmary, Oxford Rd, Manchester, UK

4. Bristol Royal Hospital for Children, Emergency Department, Bristol, UK

5. Faculty of Health and Life Sciences, University of the West of England, Bristol, UK

Social Media, Conference, Medical Education

Words: 1004

Key Points

Social Media is increasingly used to publicise and promote clinical conferences, and in particular Twitter encourages real time feedback on presentations.

There is the potential for presenters to lost control of the content of their talks or accidental facilitate the release of mis-information as there is currently virtually no guidance on social media use before or during conferences.

However the benefits in terms on focused presentations, rapid dissemination of messages and virtual attendance have also probably yet to be realised. 
The conference "hashtag" has become a regular addition to any advertising poster or programme. Social media technologies take on many different forms including internet forums, social blogs, microblogs, wikis, podcasts, and videos. It is cheap and accessible, and differentiated from 'traditional' media in quality, reach, frequency, usability, immediacy, and permanence that enable anyone (even private individuals) to publish or access information often with minimal quality assurance. The use of social media at conferences, not just confined to healthcare, has exploded. In particular Twitter has become an established tool for pre-, intra- and post-conference communication [1]. Twitter is an online social networking and microblogging service that enables users to send and read "tweets"; messages limited to 140 characters which can include pictures and photographs. Registered users can read and post tweets but unregistered users can only read them. Globally Twitter is now one of the ten most visited websites. As of June 2014, approximately 6,000 tweets are sent every second, which corresponds to over 350,000 tweets per minute, 500 million tweets per day and 200 billion tweets per year [2].

The International Conference on Emergency Medicine (ICEM) 2012 was the first significant emergency medicine conference to utilise and record twitter use with over 4500 tweets. Tweeting by participants was common, and a large number $(74.4 \%)$ of original tweets related to clinical or research material. There was also a large virtual twitter-based participation in the conference as over 400 people produced tweets about the conference, yet only $34 \%$ of those were physically present [3]. This has now been surpassed by the Social Media and Critical Care Conference (SMACCGold); over 4 days there were 23000 tweets, which have made a total of over 39 million impressions (the number of times a tweet about the conference appears across all user's timelines) [4].

There are many potential uses of Twitter at conferences, both for organisers and attendees. Pre-conference advertising, intra-conference networking and sharing of key messages from lectures and workshops are the most common. 
Whilst the use of social media including Twitter offers many potential benefits in terms of dissemination of information, sharing of new research findings and collaborative learning, there is the possibility of disparity of message (a Chinese whispers of the modern age), and issues with trustworthiness and reliability of information presented [5]. The evolving use of Twitter in scientific conferences may present opportunities for education and interaction but also may inadvertently result in misrepresentation through human error or technical restrictions (notably the limited character count of Twitter). Concerns about the use of Twitter by health care professional are already on record in respect of live tweeting from hospital settings in real-time. [6] Consideration must also be given to those that can't attend. Could the sharing of live information lead to a reduction in the number of people actually coming to conference and can those people tracking the live feed count that as Continuous Professional Development?

There may also be implications in the presentation of original research findings in relation to copyright regulations enforced by scientific journals, conflicts of interest [7] and some presenters may feel inherently uncomfortable in regards to the direct sharing of their slides using the photo sharing functions available within twitter. Despite this, the sharing of information by social media has clear potential and if the use of social media is found to be an effective means of accurately communicating messages to a wider audience, then organisers, speakers, and delegates must surely respond by assisting such communication. Conversely, if social media simply provides a well intentioned platform for the dissemination of inaccurate or incorrect information, steps must be taken to protect the integrity of scientific conferences.

Recently we assessed speakers' reactions to tweets about their talks at a national Emergency Medicine conference [8]. We found that the majority of speakers had no concerns about the content of the tweets. However 3 of 14 speakers did raise concerns that they had been mis-represented and 3 of 14 participants said they would change their approach to future presentations. These changes included making their conclusions clearer and putting up 
statements in a Twitter friendly way. This implies there is still an educational need for both speakers and conference organisers to be briefed on the potential advantage and disadvantages of twitter use. Concerns still exist as one speaker framed in the free text feedback at the end of the questionnaire.

"I think it is a useful tool principally for dissemination. Feedback is unregulated and although it can be useful, it is sometimes difficult to gauge the seniority/experience of the person commenting. I therefore welcome all opinions and comments but am unclear about how to take some of them."

The support for further study in this area was realised by another:

"Twitter is a tool for those who twitter - the many who do are outweighed by the many who don't. Does it have an effect- this needs to be determined."

There has yet to be any (known) litigation or academic conflict resulting from Twitter use at a conference but given the lack of general guidance this may an incident waiting to happen. Conversely conferences failing to embrace social media, and in particular Twitter, may find they become increasingly isolated and perceived as being of lower quality than others. Given we still have yet to determine how well do tweets accurately reflect content, whether 'tweeters' learn more than non-tweeters and whether the presenter of the future requires social media training to gain the maximal impact from their presentation we suggest the following should be considered by all conference organisers:

1. Setting rules or goodwill agreement for social media use

2. Teaching people how to utilise twitter at conferences

3. Ensuring presenters are aware of how their presentations may be utilised on social media. .

Social media has already begun to affect the content and spread of information from medical conferences. Time will tell if the construct of the traditional medical conference can keep pace with the continued rapid development of social media as a learning and knowledge dissemination tool. 


\section{References}

1. Chris Parr. Walls and Boundaries. Time Higher Education Website. http://www.timeshighereducation.co.uk/422153.article (website last accessed 5th Feburary 2015)

2. Twitter Usage Statistics. http://www.internetlivestats.com/twitterstatistics/ (website last accessed $5^{\text {th }}$ February 2015)

3. Neill A, Cronin JJ, Brannigan D, O'Sullivan R, Cadogan M. The impact of social media on a major international emergency medicine conference. Emerg Med J. 2014 May 1;31(5):401-4.

4. Symplur Statistics for \#SMACCGold http://www.symplur.com/healthcare-hashtags/smaccgold/ (website last accessed $5^{\text {th }}$ February 2015)

5. Jalali $A$ and Wood T. Tweeing during conferences: educational or just another distraction? Medical Education 2013 47;11:1129-1130

6. McCartney M. Tweets from the Emergency Department BMJ 2013; 346

7. Decamp M. Physicians, Social Media and Conflicts of Interest. J Gen Intern Med. 2013 Feb;28(2):299-303

8. Roland D, May N, Body R, Carley S and Lyttle M. Are you a SCEPTIC? SoCial mEdia Precision \& uTility In Conferences Emerg Med J doi:10.1136 